\title{
BMJ Open Cost effectiveness of screening for intracranial aneurysms among patients with bicuspid aortic valve: a Markov modelling study
}

\author{
Jigang Chen, ${ }^{1,2}$ Mingyang Han, ${ }^{3}$ Xin Feng, ${ }^{4}$ Fei Peng, ${ }^{1,2}$ Xin Tong, ${ }^{1,2}$ Hao Niu, ${ }^{1,2}$ \\ Danfeng Zhang, ${ }^{5}$ Aihua Liu (i) ${ }^{1,2}$
}

To cite: Chen J, Han M, Feng X, et al. Cost effectiveness of screening for intracranial aneurysms among patients with bicuspid aortic valve: a Markov modelling study. BMJ Open 2021;11:e051236. doi:10.1136/ bmjopen-2021-051236

- Prepublication history and additional supplemental material for this paper are available online. To view these files, please visit the journal online (http://dx.doi.org/10.1136/ bmjopen-2021-051236).

$\mathrm{JC}$ and $\mathrm{MH}$ contributed equally. $\mathrm{DZ}$ and $\mathrm{AL}$ contributed equally.

$\mathrm{JC}$ and $\mathrm{MH}$ are joint first authors.

Received 13 March 2021 Accepted 23 November 2021

Check for updates

(c) Author(s) (or their employer(s)) 2021. Re-use permitted under CC BY-NC. No commercial re-use. See rights and permissions. Published by BMJ.

For numbered affiliations see end of article.

Correspondence to

Dr Aihua Liu;

liuaihuadoctor@163.com and

Dr Danfeng Zhang;

dfzhangsmmu@163.com

\section{ABSTRACT}

Objective Bicuspid aortic valve (BAV) is common and $7.7 \%-9.8 \%$ of patients with BAV have intracranial aneuryms (IAs) which might lead to a devastating subarachnoid haemorrhage (SAH). We aimed to evaluate different screening and follow-up strategies using magnetic resonance angiography for IAs among patients with BAV.

Methods A decision-analytic model was built to evaluate the costs and effectiveness of different management strategies from the Chinese healthcare payer's perspective. The evaluated strategies included natural history without screening for possible IAs, regular screening and no follow-up for detected IAs, and regular screening with regular follow-up (Screen strategy/ Follow-up strategy). Base case calculation, as well as probabilistic, one-way, and two-way sensitivity analyses, were performed.

Results According to the base case calculation, natural history had the least cost and effectiveness while Every 5 years (y)/Annual gained the highest cost and effectiveness. Every 10y/Biennial was cost effective when compared with Every $10 y /$ Every $5 y$ under the willingness-to-pay threshold of $¥ 211743$ (US\$30162). Probabilistic sensitivity analysis showed that Every $10 \mathrm{y} /$ Biennial was superior in $88.3 \%$ of the cases when compared with Every $10 \mathrm{y} /$ Every $5 \mathrm{y}$. One-way and two-way sensitivity analyses proved that Every $10 y /$ Biennial was the dominant strategy under most circumstances.

Conclusions Screening for possible IAs among patients with BAV and follow-up for detected IAs would increase the effectiveness. Every 10y/Biennial was the optimal strategy from the Chinese healthcare payer's perspective.

\section{INTRODUCTION}

Bicuspid aortic valve (BAV) is one of the most frequent congenital heart defects with a prevalence of $1 \%-2 \%$ in the general population. ${ }^{1}$ Although patients with BAV may stay asymptomatic throughout their entire life, most suffer from an increased risk for severe cardiovascular events such as valve insufficiency, stenosis, thoracic aortic dilation or dissection..$^{2-4}$ Moreover, they are increasingly
Strengths and limitations of this study

- The 2015 American Heart Association/American Stroke Association guidelines recommended that patients with a history of bicuspid aortic valve (BAV) should be screened for intracranial aneurysms (IAs) by non-invasive computed tomographic angiography or magnetic resonance angiography (MRA). However, there are a lot of uncertainties regarding the benefits of screening for IAs in patients with BAV.

- We built a decision-analytic model to compare the costs and effectiveness of different strategies regarding the management of IAs in patients with BAV. Our model suggested that a repeated screen with MRA every 10 years was optimal. Once IAs were detected, the large ones could be coiled directly while the small ones could have a biennial follow-up for IAs growth. Our study provides a reference for the management of these patients.

- Some key variables including the risk ratio of rupturing and growing in large IAs compared with small IAs were not retrieved from BAV-related studies due to lack of literature.

- There were no Chinese population-specific clinical parameters and utilities, and most of these values were obtained from international studies. This might cause some bias towards our results, especially from the Chinese perspective.

- We do not consider the effect of endovascular complications on the cost effectiveness of different management strategies since there were no reliable sources to estimate the incidence and costs.

vulnerable to intracranial aneurysms (IAs) formation. The prevalence of IAs among patients with BAV is significantly higher than the general population: $7.7 \%-9.8 \%$ versus $3.2 \%$, respectively. ${ }^{5-7}$

IAs might lead to a catastrophic consequence due to subarachnoid haemorrhage (SAH) caused by aneurysm rupture. Therefore, screening populations with an increased risk of IAs formation would be beneficial due to the prophylactic treatment. For example, 
with a high prevalence of $11.5 \%,{ }^{8}$ systematic screening for IAs in autosomal dominant polycystic kidney disease was found to increase quality-adjusted life-years (QALYs) and be cost effective. ${ }^{910}$

The 2015 American Heart Association/American Stroke Association guidelines recommended that patients with a BAV history should be screened for IAs by non-invasive computed tomographic angiography or magnetic resonance angiography (MRA). ${ }^{11}$ However, since screening acquired extra expenditure and prophylactic coiling or open surgery might bring unpleasant consequences including disability or even death, there are a lot of uncertainties regarding the benefits of screening for IAs in patients with BAV. The frequency for IAs screening, as well as the optimal follow-up strategy once IA was detected, have not been determined. In this study, we built a decision-analytic model to compare the costs and effectiveness of different management strategies using MRA in patients with BAV.

\section{METHODS}

Natural history without screening was compared with different strategies including one-time screening for possible IAs among patients with BAV at the initial age, screening every 10 years, and screening every 5 years. The Second International Study of Unruptured Intracranial Aneurysms indicated that the risk of bleeding from UIAs less than $7 \mathrm{~mm}$ was extremely low. ${ }^{12}$ Therefore, once large IAs (size of more than $7 \mathrm{~mm}$ ) were detected by screening, they were assumed to have endovascular treatment directly. For small IAs with a size of less than $7 \mathrm{~mm}$, they would be followed regularly to assess the possible growth in size because growing IAs were more likely to rupture. ${ }^{13}$ The follow-up strategies included no follow-up, follow-up every 5 years, biennial follow-up and annual follow-up. To make the following descriptions more simplified, the management strategy was expressed as 'Screen strategy/ Follow-up strategy'. For example, screen every 10 years followed by annual follow-up would be 'Every 10y/ Annual'. We conducted this study according to the Consolidated Health Economic Evaluation Reporting Standards (CHEERS) reporting guideline and the CHEERS list was provided in online supplemental table.

\section{Markov model}

A decision-analytic tree incorporating Markov modelling was built by TreeAge Pro Suite 2020 (TreeAge Software). It started with a 30-year-old patient with BAV, representing the age he/she entered the cohort. This model used 1 year as a cycle and would repeat until all patients died or reached 99 years old. Thirteen different discrete health states were defined in this model. The Markov model is presented in online supplemental figure 1.

\section{Natural history}

If no screening was performed for patients with $\mathrm{BAV}, 8 \%$ of them were assumed to have underlying IAs according to a recent meta-analysis. ${ }^{14}$ All these IAs carried an annual risk of SAH due to aneurysm rupture. We assumed all patients with aneurysmal SAH would be presented to endovascular coiling. According to the treatment outcomes, these patients might have a full recovery, survive with mild disability, survive with moderate to severe disability, or die. After treatment, patients with a full recovery would receive annual MRA follow-up in subsequent years due to de novo aneurysm formation.

For those without IAs, there were no associated costs or reduction of utility in their health state. However, they would have an annual incidence of de novo aneurysm formation. Once a new aneurysm was formed, it also carried a risk of rupturing.

\section{Screening without follow-up}

In this strategy, patients with BAV were assumed to receive one-time or regular screening for possible IAs. Since large IAs had a significantly higher risk of rupturing than the small ones. ${ }^{1516}$ We assumed patients with large IAs would have prophylactic coiling once detected by screening. After treatment, these patients would have a full recovery, mild disability, moderate to severe disability, or death. Fully recovered patients would have a similar subsequent follow-up as in 'No screening'.

If small IAs were detected by screening, no follow-up would be performed. These patients would also have a risk of rupturing and subsequent events as described in "Natural history".

If no IAs were detected among patients with BAV, they still had a risk of de novo aneurysm formation and then aneurysm rupture. For the unruptured new aneurysm, it would not be detected until the next screening.

\section{Screening with follow-up}

Similar to 'Screening without follow-up', patients with BAV would receive one-time or regular screening and large detected IAs would be coiled directly. For small detected IAs, they would undergo different follow-up strategies with regular intervals to assess the possible growth in aneurysmal size. If growth was detected, patients would have direct coiling with similar subsequent outcomes and follow-up described in 'Screening without follow-up'. If there was no size change in follow-up, IAs still had a smaller rupture risk.

\section{Clinical parameters}

All the clinical parameters used in the model were retrieved from the published large-cohort studies or reviews whenever possible (table 1 ). The proportion of IAs with a size of less than $7 \mathrm{~mm}$ was calculated from the recent cohort study conducted by Egbe et al in which $84.7 \%$ was small. ${ }^{5}$ The annual incidence of de novo IAs formation among patients with BVA could not be determined by the current literature. Pickard et al calculated an annual incidence of $0.439 \%$ for IAs formation among patients with coarctation of the aorta in their study. ${ }^{17}$ Since BAV and coarctation are two closely related aortopathies, ${ }^{18}$ 
Table 1 List of input variables

\begin{tabular}{|c|c|c|c|c|}
\hline Variable & Mean & Range & Distribution & Reference \\
\hline \multicolumn{5}{|l|}{ Clinical parameters } \\
\hline IAs prevalence at the initial screening & 0.08 & $0.06-0.10$ & $\begin{array}{l}\text { Beta } \\
\text { SD: } 0.006\end{array}$ & 14 \\
\hline Proportion of small IAs & 0.847 & $0.756-0.939$ & $\begin{array}{l}\text { Beta } \\
\text { SD: } 0.04\end{array}$ & 5 \\
\hline Annual incidence of de novo IAs formation & 0.004 & $0.002-0.007$ & $\begin{array}{l}\text { Beta } \\
\text { SD: } 0.001\end{array}$ & 17 \\
\hline Rupture rate of small IAs & 0.004 & $0.001-0.009$ & $\begin{array}{l}\text { Beta } \\
\text { SD: } 0.001\end{array}$ & 515 \\
\hline Rupture rate of large IAs & 0.017 & $0.001-0.04$ & $\begin{array}{l}\text { Beta } \\
\text { SD: } 0.007\end{array}$ & 515 \\
\hline Risk ratio of growing in large IAs compared with small IAs & 3.68 & & & 19 \\
\hline Risk ratio of rupturing in growing IAs compared with nongrowing ones & 12 & $\pm 20 \%$ & - & 13 \\
\hline Proportion of mild disability after coiling of unruptured IAs & 0.048 & $0.034-0.065$ & $\begin{array}{l}\text { Beta } \\
\text { SD: } 0.005\end{array}$ & 1620 \\
\hline Proportion of moderate to severe disability after coiling of unruptured IAs & 0.022 & $0.013-0.034$ & $\begin{array}{l}\text { Beta } \\
\text { SD: } 0.004\end{array}$ & 1620 \\
\hline Mortality after coiling of unruptured IAs & 0.006 & $0.002-0.01$ & $\begin{array}{l}\text { Beta } \\
\text { SD: } 0.001\end{array}$ & 1620 \\
\hline Proportion of mild disability after coiling of ruptured IAs & 0.15 & $0.13-0.17$ & $\begin{array}{l}\text { Beta } \\
\text { SD: } 0.007\end{array}$ & $21-24$ \\
\hline \multicolumn{5}{|l|}{ Cost $(¥)$} \\
\hline MRA & 600 & $480-720$ & $\begin{array}{l}\text { Normal } \\
\text { SD: } 60\end{array}$ & 28 \\
\hline Coiling & 160646 & $128517-192775$ & $\begin{array}{l}\text { Gamma } \\
\text { SD: } 64828\end{array}$ & 28 \\
\hline SAH treatment & 3829 & 3063-4595 & $\begin{array}{l}\text { Gamma } \\
\text { SD: } 586\end{array}$ & 28 \\
\hline Annual cost of mild disability & 9108 & 8826-9397 & $\begin{array}{l}\text { Gamma } \\
\text { SD: } 143\end{array}$ & 29 \\
\hline Annual cost of moderate to severe disability & 13999 & $13234-14796$ & $\begin{array}{l}\text { Gamma } \\
\text { SD: } 391\end{array}$ & 29 \\
\hline \multicolumn{5}{|l|}{ Utility } \\
\hline BAV & 0.92 & $0.89-0.94$ & Triangle & 31 \\
\hline Mild disability & 0.72 & $0.65-0.80$ & Triangle & 21 \\
\hline Moderate to severe disability & 0.41 & $0.25-0.65$ & Triangle & 21 \\
\hline $\mathrm{SAH}$ & 0.64 & $0.52-0.71$ & Triangle & 42 \\
\hline Coiling procedure & $5 \%$ disutility & & & 9 \\
\hline
\end{tabular}

BAV, bicuspid aortic valve; IA, intracranial aneurysm; MRA, magnetic resonance angiography; SAH, subarachnoid haemorrhage. 
and patients with coarctation have a slightly higher prevalence of IAs than those with $\mathrm{BAV},{ }^{14}$ we used a lower annual incidence of $0.4 \%$ for the base case calculation and a range of $0.2 \%-0.7 \%$ for sensitivity analyses in our study after consulting with the experts. The rupture rate of IAs in patients with BAV was $4 \%$ over 7 years. ${ }^{5}$ The risk ratio of rupturing in large IAs compared with small ones was 4.6. ${ }^{15}$ We then calculated the rupture rate of small and large IAs to be $0.4 \%$ and $1.7 \%$, respectively. Egbe $e t$ al reported an overall IAs growth rate of $6 \%$ over 9 years in patients with BAV. ${ }^{5}$ The risk ratio of growing in large IAs compared with small ones was estimated to be $3.68{ }^{19}$ Then, the annual growth rate of small and large IAs in patients with BAV was calculated to be $0.5 \%$ and $1.8 \%$, respectively. There was a 12-fold higher risk of rupturing for growing IAs compared with nongrowing ones. ${ }^{13}$ The outcomes of prophylactic coiling were combined from a large cohort study and a meta-analysis. ${ }^{16}{ }^{20}$ The longterm case fatality after SAH treatment was estimated to be $35 \%$, while $15 \%$ and $9 \%$ of them would develop mild or moderate to severe disability. ${ }^{21-24}$

The age-specific mortality rates were obtained from the most recent published census of China and were adjusted by the aneurysmal cause of death in China. ${ }^{25}{ }^{26}$ Patients with disabilities would have increased mortality compared with the general population. The mortality rate for mild disability was adjusted by 2.02-fold, and for moderate to severe disability was adjusted by 4.46 -fold. ${ }^{27}$

\section{Costs}

This study was conducted from the Chinese healthcare payers' perspective and the costs included both out-ofpocket costs and reimbursements. Costs of MRA, endovascular treatment, SAH, and post-hospitalisation care were extracted from Chinese-based studies and adjusted from years of publication. ${ }^{28}{ }^{29}$ They were updated to the 2019 Chinese Yuan (¥) using the medical care component of the consumer price index. For example, in our previous cost-effectiveness analysis, the cost of MRA was reported to be $¥ 600$ which was obtained from the clinical database of our hospital. ${ }^{28}$ This cost was standardised to the 2019 price in this study and we, therefore, used this value directly. Most of the costs were assigned with Gamma distributions since they were rightward skewed with a lower boundary of zero, which is very useful for varying cost inputs in the probabilistic sensitivity analysis. ${ }^{30}$

\section{Utilities}

Health-related quality of life value (utility score) was assigned to all the health states. QALYs were measured to determine health outcomes by multiplying the length of patient years within a particular health state by the corresponding utility score. Patients with BAV were assumed to have a utility of $0.92 .{ }^{31}$ The utility of mild disability, moderate to severe disability, and SAH were based on a previous cost-effectiveness analysis of the preventive treatment of unruptured aneurysms. ${ }^{21}$ The coiling procedure was assumed to cause a temporary $5 \%$ disutility due to discomfort or anxiety. ${ }^{9}$ All the costs and utilities were applied with a $3 \%$ discount rate annually.

\section{Statistical analysis}

All the analyses were conducted by TreeAge Pro Suite 2020. We calculated the incremental cost-effectiveness ratio (ICER) defined as follows: (Cost of Strategy 1 - Cost of reference strategy)/(QALYs of Strategy 1 - QALYs of reference strategy). A strategy was cost effective if the ICER was less than the willingness-to-pay threshold (WTP) of $3 \times$ gross domestic product (GDP) per capita in that country. ${ }^{32}$ The GDP per capita of China in 2019 was $¥ 70581$ and equaled US $\$ 10054 .{ }^{33}$ Therefore, we used a WTP threshold of $¥ 211743$ (US\$30 162) per QALY in this study. Another primary indicator was the net monetary benefit, which was defined as follows: expected utility $\times$ WTP - cost. A strategy was optimal if it had a higher net monetary benefit than other ones.

A half-cycle correction was applied in this study. The base case calculation was performed with the mean value of each parameter. To account for the uncertainty of specific parameters on the model outcome, one-way and two-way sensitivity analyses were performed by varying parameters in their ranges while keeping other parameters fixed. Probabilistic sensitivity analysis with Monte Carlo simulation was carried out with all input parameters varied simultaneously according to their assigned distributions. We calculated the probability of cost effectiveness for each strategy by varying WTP values using cost-effectiveness acceptability curves (CEAC).

\section{Patient and public involvement}

Patients and/or the public were not involved in the study.

\section{RESULTS}

\section{Base case calculation}

The cost and effectiveness of different strategies were compared (figure 1). According to the results, natural history had the least cost but also the least effectiveness. Every 5y/Annual gained the highest cost as well

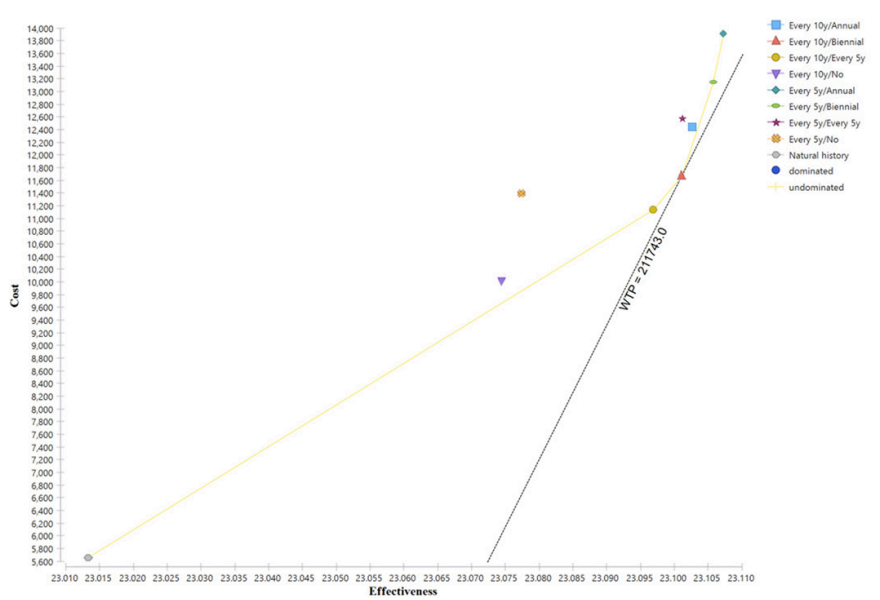

Figure 1 The cost and effectiveness of different management strategies. WTP, willingness to pay. 
Table 2 Base case calculation (dominated strategies have been removed)

\begin{tabular}{lclllc} 
Different strategies & Cost $(¥)$ & Effectiveness (QALY) & Incremental cost (¥) & $\begin{array}{l}\text { Incremental } \\
\text { effectiveness (QALY) }\end{array}$ & $\begin{array}{l}\text { ICER (¥/ } \\
\text { QALY) }\end{array}$ \\
\hline Natural history & 5659 & 23.013 & Reference & Reference & Reference \\
Every 10y/Every 5y & 11135 & 23.097 & 5476 & 0.084 & 65414 \\
Every 10y/Biennial $^{*}$ & 11673 & 23.101 & 537 & 0.004 & 129354 \\
Every 5y/Biennial $^{*}$ & 13142 & 23.106 & 1469 & 0.005 & 314778 \\
Every 5y/Annual $^{*}$ & 13926 & 23.107 & 784 & 0.002 & 488453 \\
\hline
\end{tabular}

*ICER larger than WTP threshold.

ICER, incremental cost-effectiveness ratio; QALY, quality-adjusted life years; WTP, willingness to pay.

as the highest effectiveness. The yellow line in figure 1 that connected five different strategies was the costeffectiveness frontier. Strategies located on the left of the frontier were not cost-effective compared with those on the frontier. We then calculated the ICERs using only the strategies on the cost-effectiveness frontier (table 2). Every 10y/Biennial had an ICER of ¥129 354/QALY when compared with Every 10y/Every 5y and it was cost effective under the WTP threshold of $¥ 211$ 743/QALY.

\section{Probabilistic sensitivity analyses}

Probabilistic sensitivity analysis was performed with 10000 iterations. When compared with Every 10y/Every $5 y$, Every 10y/Biennial was superior in 8830 of the 10000 iterations $(88.3 \%$ ) (figure 2 ). The result remained stable after 10 repeated analyses, indicating that these iterations were sufficient to achieve a reliable outcome.

The CEAC showing the cost-effective strategy depending on the WTP threshold is presented in figure 3. The probability of Every 10y/Biennial being cost-effective would be greater than all other strategies when the WTP is above $¥ 129354$ /QALY, which is lower than the current threshold of $¥ 211743$ /QALY.



Figure 2 Probabilistic sensitivity analysis. Each dot on the diagram represents one of the 10000 iterations. All dots below the line of WTP represent iterations in which Every 10y/ Biennial is superior to Every 10y/Every 5y. WTP, willingness to pay.

\section{One-way and two-way sensitivity analyses}

A tornado diagram is a set of one-way sensitivity analyses brought together in a single graph. As presented in figure 4, the optimal management strategy would change along with the variations of the rupture rate of small IAs, the annual incidence of de novo IAs formation, the growth rate of small IAs, and the rupture rate of large IAs. We put these four parameters in the oneway sensitivity analyses. The result showed that Every 10y/ Biennial was the optimal strategy when the rupture rate of small IAs was above $0.26 \%$ even though the difference was small (online supplemental figure 2). For the annual incidence of de novo IAs formation, Every10y/Biennial was dominant when the incidence was less than $0.65 \%$. Similarly, when the rate was above $0.28 \%$ for the growth rate of small IAs and was between $0.3 \%$ and $2.85 \%$ for the rupture rate of large IAs, Every 10y/Biennial remained optimal.

Then, we performed two-way sensitivity analyses on the rupture rate of small IAs and the rupture rate of large IAs, as well as on the annual incidence of de novo IAs formation and the growth rate of small IAs. The results indicated that Every 10y/Biennial was the optimal strategy under most circumstances (online supplemental figure

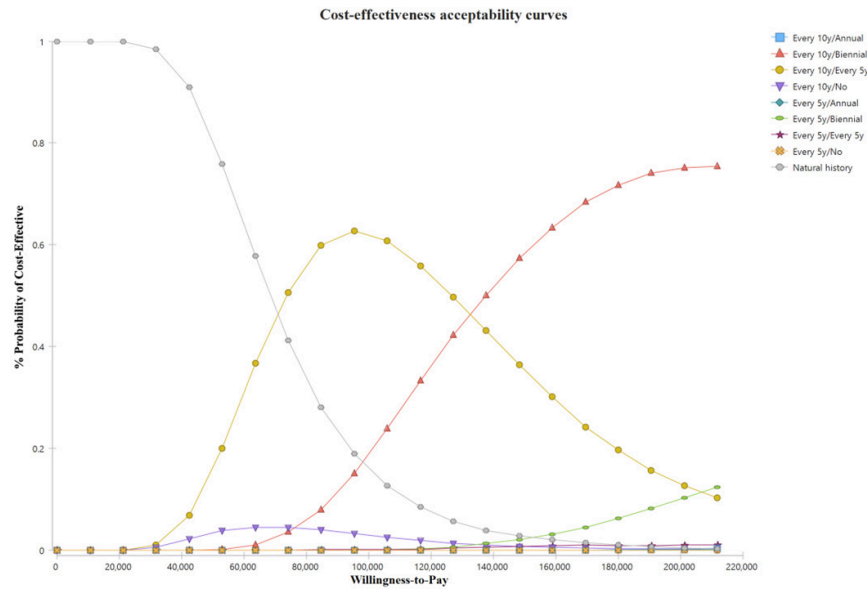

Figure 3 Cost-effectiveness acceptability curve. The probability of cost effectiveness for each strategy under a given willingness-to-pay threshold was presented. The result was from 10000 iterations of the Markov model. 


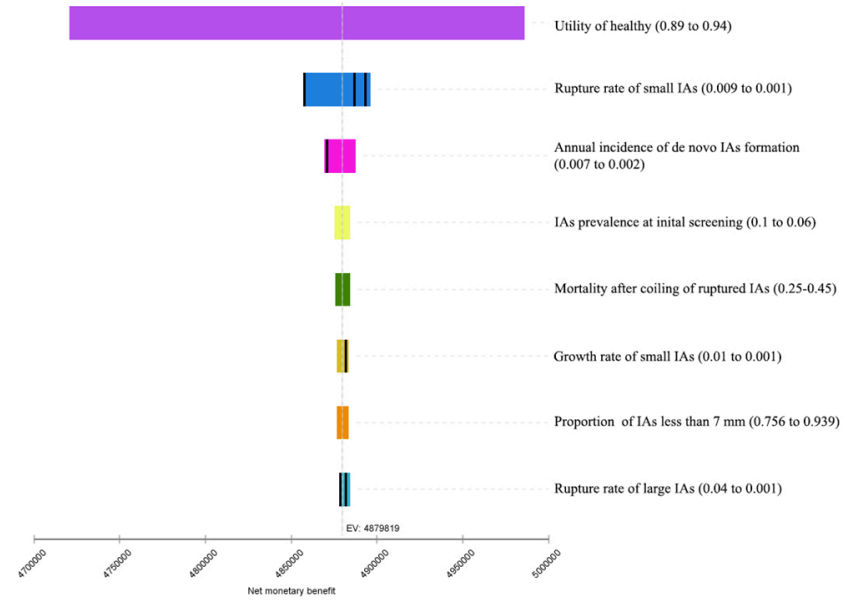

Figure 4 Tornado diagram. The effect of variation of each parameter on the net monetary benefit was presented on each bar. The dark line within the bar represented the alteration of the optimal strategy. EV, expected value; IA, intracranial aneurysm.

3), IV). We also included the age of starting screening (from 30 to 50 years old) and IAs prevalence at the initial screening in the two-way sensitivity analysis and the results indicated that the optimal strategy was not sensitive to these two parameters (online supplemental figure 4).

\section{DISCUSSION}

The reported prevalence of IAs in patients with BAV was estimated to be $7.7 \%-9.8 \%,{ }^{5}{ }^{6}$ which was significantly higher than that among the general population. Since there is no direct evidence or consensus among experts, a decision analysis may help decide the best management strategy for these patients. To the best of our knowledge, this is the first study that has evaluated the costs and utility of screening and following for IAs in patients with BAV. Our study supported the recommendation from the 2015 American Heart Association/American Stroke Association guidelines that patients with BAV should be screened and followed regularly. ${ }^{11}$ The results showed that MRA screening patients with BAV every 10 years, and biennial follow-up for the detected small IA, was the optimal strategy from the Chinese healthcare payer's perspective. This strategy remained optimal when the age starting screen ranged between 30 to 50 years old.

China has the largest population around the world and there are fast increasing demands for limited healthcare budgets. It drives policy-makers to move towards a data-driven and evidence-supporting healthcare system with China's national health strategy. ${ }^{34}$ Actually, several health policy reforms have advocated comparative cost and effectiveness researches in recent years. ${ }^{35}$ The number of published cost-effectiveness analyses in China has grown rapidly over the last two decades. ${ }^{36}$ Our study has the merits of providing an evidence-based suggestion regarding the management of patients with BAV, which has a high prevalence among the general population. ${ }^{37}$
We conducted one-way sensitivity analyses using all the input parameters to determine the impact of parameter uncertainty on the primary outcome. The variations of four parameters including the rupture rate of small IAs, the annual incidence of de novo IAs formation, the growth rate of small IAs, and the rupture rate of large IAs would affect our results. The mean value of the rupture rate of small $(0.4 \%)$ and large IAs $(1.7 \%)$ was calculated from the largest cohort study related to IAs in patients with $\mathrm{BAV}^{5}{ }^{5}$ These rates were similar to those reported in the aneurysm cohorts among the general population. ${ }^{15} 16$ The growth rate of small IAs $(0.5 \%)$ was also calculated from this cohort study. ${ }^{5}$ The annual incidence of de novo IAs formation among patients with BAV was another key parameter while its value could not be obtained directly from the published literature. In a recent meta-analysis, the incidence of de novo IAs formation was estimated to be $0.3 \%$ among patients with a prior diagnosis of IAs. ${ }^{38}$ In another similar cost-effectiveness study, Pickard et al calculated an annual incidence of $0.439 \%$ among patients with aorta coarctation. ${ }^{17}$ Therefore, we used $0.4 \%$ for the annual incidence of IA formation in the current study. The ranges assigned to these parameters were estimated after consulting with experts based on the related literature and we tend to use a generally wider range to account for the uncertainty if possible.

After varying these four aforementioned parameters in the two-way sensitivity analyses, Every 10y/Biennial remained the optimal strategy under most circumstances. This result was further validated by the probabilistic sensitivity analysis. Moreover, we calculated the probability of cost effectiveness for each strategy by varying WTP values and the result indicated that Every $10 \mathrm{y} /$ Biennial was more likely to be cost-effective than all other strategies together under the WTP threshold of ¥211743/QALY. Taking all these results together, screening for IAs among patients with BAV every 10 years with biennial follow-up for detected small IAs was the more cost-effective strategy currently.

Our study has several limitations. First, some key variables including the risk ratio of rupturing and growing in large IAs compared with small IAs were not retrieved from BAV related studies due to lack of literature. We used the value from pooled study and meta-analysis that targeted IAs among the general population instead. There were no Chinese population-specific clinical parameters and utilities, and most of these values were obtained from international studies. This might cause some bias towards our results, especially from the Chinese perspective. However, the sensitivity analyses have accounted for these uncertainties and the model conclusion remained unchanged within the ranges of these parameters. Moreover, this is not unprecedented in other cost-effectiveness studies. ${ }^{9}{ }^{17}$

Second, a constant rupture rate was assumed for both large and small IAs. It has been postulated that the rupture risk is higher shortly after formation and the risk might decline with time. ${ }^{39}$ This unconstant rupture rate might imply closer supervision of these IAs initially, 
with increasing space of imaging over time. However, there lacks specific literature on the dynamic rupture risk pattern of IAs. An updated model might be needed once these data are available.

Third, we used MRA as the screening modality for IAs concerning the risk brought by contrast material and radiation of CT angiography. We assumed that each aneurysm could be detected by MRA. However, some studies do have concerns about the sensitivity and specificity of MRA for the detection of IAs, especially for the small ones. ${ }^{40}$ Moreover, there lacks published literature regarding the accuracy of detecting aneurysm growth, ${ }^{41}$ and the definition of growth is varied across different studies.

Fourth, we do not consider the effect of endovascular complications on the cost-effectiveness of different management strategies since there were no reliable sources to estimate the incidence and costs. However, considering that the fact the incidence is relatively low, it will not affect our results to a large extent.

\section{CONCLUSION}

Our study supports the recommendation to screen for possible IAs among patients with BAV. Screening and follow-up would increase the effectiveness. From the Chinese healthcare payer's perspective, a repeated screen with MRA every 10 years was suggested. Once IAs were detected, the large ones could be coiled directly while the small ones could have a biennial follow-up for IAs growth.

\section{Author affiliations \\ ${ }^{1}$ Beijing Neurosurgical Institute, Capital Medical University, Beijing, China \\ ${ }^{2}$ Department of Interventional Neuroradiology, Beijing Tiantan Hospital, Capital \\ Medical University, Beijing, China \\ ${ }^{3}$ Department of Neurosurgery, The Third Xiangya Hospital of Central South \\ University, Changsha, Hunan, China \\ ${ }^{4}$ Department of Neurosurgery, Beijing Hospital, Beijing, China \\ ${ }^{5}$ Department of Neurosurgery, Naval Medical University, Shanghai, China}

Acknowledgements We would like to express our gratitude to Yuesong Pan, an expert who has 6 years of performing cost-effectiveness, for his work in reviewing and validating the model structure, data source, statistical analyses, and results. We would also like to thank Yuesong Pan and Zhifei Wang for providing input on likely ranges of clinical parameters.

Contributors JC designed the study and built the model. DZ and MH prepared the literature and input variables. XF, FP, HN and XT conducted the statistical analysis. MH prepared the manuscript. AL prepared the manuscript and received the funding. DZ prepared the manuscript. JC, MH and HN reviewed and validated the model structure, data source, statistical analyses and results. AL and DZ had full access to all data in the study, had final responsibility for the decision to submit for publication and are responsible for the overall content as the guarantor.

Funding This study is funded by the Beijing Science and Technology Planning Project (No. Z181100009618035).

Competing interests None declared.

Patient consent for publication Not applicable.

Ethics approval Ethical approval from the institutional review board was waived because no human subjects were involved in this study.

Provenance and peer review Not commissioned; externally peer reviewed.

Data availability statement All data relevant to the study are included in the article or uploaded as supplementary information. Data are available upon reasonable request.
Supplemental material This content has been supplied by the author(s). It has not been vetted by BMJ Publishing Group Limited (BMJ) and may not have been peer-reviewed. Any opinions or recommendations discussed are solely those of the author(s) and are not endorsed by BMJ. BMJ disclaims all liability and responsibility arising from any reliance placed on the content. Where the content includes any translated material, BMJ does not warrant the accuracy and reliability of the translations (including but not limited to local regulations, clinical guidelines, terminology, drug names and drug dosages), and is not responsible for any error and/or omissions arising from translation and adaptation or otherwise.

Open access This is an open access article distributed in accordance with the Creative Commons Attribution Non Commercial (CC BY-NC 4.0) license, which permits others to distribute, remix, adapt, build upon this work non-commercially, and license their derivative works on different terms, provided the original work is properly cited, appropriate credit is given, any changes made indicated, and the use is non-commercial. See: http://creativecommons.org/licenses/by-nc/4.0/.

ORCID iD

Aihua Liu http://orcid.org/0000-0002-6391-805X

\section{REFERENCES}

1 Ward C. Clinical significance of the bicuspid aortic valve. Heart 2000;83:81-5.

2 Michelena HI, Desjardins VA, Avierinos J-F, et al. Natural history of asymptomatic patients with normally functioning or minimally dysfunctional bicuspid aortic valve in the community. Circulation 2008;117:2776-84.

3 Tzemos N, Therrien J, Yip J, et al. Outcomes in adults with bicuspid aortic valves. JAMA 2008;300:1317-25.

4 Niaz T, Fernandes SM, Sanders SP, et al. Clinical history and management of bicuspid aortic valve in children and adolescents. Prog Cardiovasc Dis 2020;63:425-33.

5 Egbe AC, Padang R, Brown RD, et al. Prevalence and predictors of intracranial aneurysms in patients with bicuspid aortic valve. Heart 2017;103:1508-14.

6 Schievink WI, Raissi SS, Maya MM, et al. Screening for intracranial aneurysms in patients with bicuspid aortic valve. Neurology 2010;74:1430-3.

7 Vlak MH, Algra A, Brandenburg R, et al. Prevalence of unruptured intracranial aneurysms, with emphasis on sex, age, comorbidity, country, and time period: a systematic review and meta-analysis. Lancet Neurol 2011;10:626-36.

8 Cagnazzo F, Gambacciani C, Morganti R, et al. Intracranial aneurysms in patients with autosomal dominant polycystic kidney disease: prevalence, risk of rupture, and management. A systematic review. Acta Neurochir 2017;159:811-21.

9 Malhotra A, Wu X, Matouk CC, et al. Mr angiography screening and surveillance for intracranial aneurysms in autosomal dominant polycystic kidney disease: a cost-effectiveness analysis. Radiology 2019;291:400-8.

10 Flahault A, Trystram D, Nataf F, et al. Screening for intracranial aneurysms in autosomal dominant polycystic kidney disease is costeffective. Kidney Int 2018;93:716-26.

11 Thompson BG, Brown RD, Amin-Hanjani S, et al. Guidelines for the management of patients with unruptured intracranial aneurysms: a guideline for healthcare professionals from the American heart Association/American stroke association. Stroke 2015;46:2368-400.

12 Wiebers DO, ISoUIA I. Unruptured intracranial aneurysms: natural history, clinical outcome, and risks of surgical and endovascular treatment. The Lancet 2003;362:103-10.

13 Villablanca JP, Duckwiler GR, Jahan R, et al. Natural history of asymptomatic unruptured cerebral aneurysms evaluated at CT angiography: growth and rupture incidence and correlation with epidemiologic risk factors. Radiology 2013;269:258-65.

14 Yu X, Xia L, Jiang Q, et al. Prevalence of intracranial aneurysm in patients with Aortopathy: a systematic review with meta-analyses. $J$ Stroke 2020;22:76-86.

15 Greving JP, Wermer MJH, Brown RD, et al. Development of the phases score for prediction of risk of rupture of intracranial aneurysms: a pooled analysis of six prospective cohort studies. Lancet Neurol 2014;13:59-66.

16 Wiebers DO, Whisnant JP, Huston J, et al. Unruptured intracranial aneurysms: natural history, clinical outcome, and risks of surgical and endovascular treatment. Lancet 2003;362:103-10.

17 Pickard SS, Prakash A, Newburger JW, et al. Screening for intracranial aneurysms in coarctation of the aorta: a decision 
and cost-effectiveness analysis. Circ Cardiovasc Qual Outcomes 2020;13:e006406.

18 Siu SC, Silversides CK. Bicuspid aortic valve disease. J Am Coll Cardiol 2010;55:2789-800.

19 Brinjikji W, Zhu Y-Q, Lanzino G, et al. Risk factors for growth of intracranial aneurysms: a systematic review and meta-analysis. AJNR Am J Neuroradiol 2016;37:615-20.

20 Lanterna LA, Tredici G, Dimitrov BD, et al. Treatment of unruptured cerebral aneurysms by embolization with guglielmi detachable coils: case-fatality, morbidity, and effectiveness in preventing bleeding--a systematic review of the literature. Neurosurgery 2004;55:767-78.

21 Greving JP, Rinkel GJE, Buskens E, et al. Cost-Effectiveness of preventive treatment of intracranial aneurysms: new data and uncertainties. Neurology 2009;73:258-65.

22 Lindgren A, Vergouwen MDI, van der Schaaf I, et al. Endovascular coiling versus neurosurgical clipping for people with aneurysmal subarachnoid haemorrhage. Cochrane Database Syst Rev 2018;8:CD003085.

23 Nieuwkamp DJ, Setz LE, Algra A, et al. Changes in case fatality of aneurysmal subarachnoid haemorrhage over time, according to age, sex, and region: a meta-analysis. Lancet Neurol 2009;8:635-42.

24 Molyneux AJ, Kerr RSC, Yu L-M, et al. International subarachnoid aneurysm trial (ISAT) of neurosurgical clipping versus endovascular coiling in 2143 patients with ruptured intracranial aneurysms: a randomised comparison of effects on survival, dependency, seizures, rebleeding, subgroups, and aneurysm occlusion. Lancet 2005;366:809-17.

25 Wang W, Jiang B, Sun H, et al. Prevalence, Incidence, and Mortality of Stroke in China: Results from a Nationwide Population-Based Survey of 480687 Adults. Circulation 2017;135:759-71.

26 National Bureau of Statistics of China. The 2010 population census of the peaple's republic of china. Available: http://www.stats.gov.cn/ tjsj/pcsj/rkpc/6rp/indexch.htm [Accessed 25 Feb 2016].

27 Ronkainen A, Niskanen M, Rinne J, et al. Evidence for excess long-term mortality after treated subarachnoid hemorrhage. Stroke 2001;32:2850-3.

28 Chen J, Feng X, Peng F, et al. Cost-Effective analysis of different diagnostic strategies in screening for aneurysms after spontaneous subarachnoid hemorrhage. Acad Radiol 2020. doi:10.1016/j. acra.2020.11.021. [Epub ahead of print: 04 Dec 2020].
29 Pan Y, Cai X, Huo X, et al. Cost-Effectiveness of mechanical thrombectomy within 6 hours of acute ischaemic stroke in China. BMJ Open 2018;8:e018951.

30 Briggs $\mathrm{AH}$, Weinstein MC, Fenwick EAL, et al. Model parameter estimation and uncertainty analysis: a report of the ISPOR-SMDM modeling good research practices Task force working Group-6. Med Decis Making 2012;32:722-32.

31 Berghammer M, Karlsson J, Ekman I, et al. Self-Reported health status (EQ-5D) in adults with congenital heart disease. Int $J$ Cardiol 2013;165:537-43.

32 World Health Organization. Macroeconomics and health: investing in health for economic development. Report of the Commission on Macroeconomics and health. Geneva: World Health Organization, 2001. http://apps.who.int/iris/bitstream/10665/42435/1/924154550X. pdf

33 National Bureau of statistics of China (in Chinese), 2019. Available: https://data.stats.gov.cn/easyquery.htm?cn=C01\&zb=A0201\&sj=2019

34 Tan X, Liu X, Shao H. Healthy China 2030: a vision for health care. Value Health Reg Issues 2017;12:112-4.

$35 \mathrm{Hu}$ J, Mossialos E. Pharmaceutical pricing and reimbursement in China: when the whole is less than the sum of its parts. Health Policy 2016;120:519-34.

36 Butt T, Liu GG, Kim DD, et al. Taking stock of cost-effectiveness analysis of healthcare in China. BMJ Glob Health 2019;4:e001418.

37 Wang $X$, Wang W. Prevalence of bicuspid aortic valve in Chinese patients with aortic valve disease: a systematic review. J Heart Valve Dis 2017;26:274-80.

38 Giordan E, Lanzino G, Rangel-Castilla L, et al. Risk of de novo aneurysm formation in patients with a prior diagnosis of ruptured or unruptured aneurysm: systematic review and meta-analysis. $J$ Neurosurg 2018;131:14-24.

39 Sato K, Yoshimoto Y. Risk profile of intracranial aneurysms: rupture rate is not constant after formation. Stroke 2011;42:3376-81.

40 Rustemi O, Alaraj A, Shakur SF, et al. Detection of unruptured intracranial aneurysms on noninvasive imaging. is there still a role for digital subtraction angiography? Surg Neurol Int 2015;6:175.

41 Malhotra A, Wu X, Geng B, et al. Management of small unruptured intracranial aneurysms: a survey of Neuroradiologists. AJNR Am J Neuroradiol 2018;39:875-80.

42 Bor ASE, Koffijberg H, Wermer MJH, et al. Optimal screening strategy for familial intracranial aneurysms: a cost-effectiveness analysis. Neurology 2010;74:1671-9. 